\title{
Determinação da atividade antiparasitária de plantas medicinais frente a parasitas gastrointestinais
}

\section{Determination of antiparasitic actividy of medicinal plants Against gastrointestinal parasites}

DOI: $10.46919 / \operatorname{archv2n5-002~}$

Recebimento dos originais: 01/05/2021

Aceitação para publicação: 31/06/2021

\section{Kellvin Costa Maciel}

Discente de Farmácia pelo Centro Universitário Tabosa de Almeida Asces-Unita, Caruaru, Pernambuco, Brasil.

\section{Nayane Monalys Silva de Lima}

Farmacêutica formado pelo Centro Universitário Tabosa de Almeida Asces-Unita, Caruaru, Pernambuco, Brasil.

\section{Cícero Jádson da Costa}

Biomédico formado pelo Centro Universitário Tabosa de Almeida Asces-Unita, Caruaru, Pernambuco, Brasil

\section{Carlos Alberto Mendes da Silva Filho}

Biomédico formado pelo Centro Universitário Tabosa de Almeida Asces-Unita, Caruaru, Pernambuco, Brasil

\section{Josenildo Severino de Vasconcelos}

Discente de Biomedicina pelo Centro Universitário Tabosa de Almeida Asces-Unita, Caruaru, Pernambuco, Brasil.

\section{Jose Tarso Gabriel de Oliveira e Sousa}

Discente de Farmácia pelo Centro Universitário Tabosa de Almeida Asces-Unita, Caruaru, Pernambuco, Brasil.

\section{Iris Paula de Araujo Torres}

Discente de Farmácia pelo Centro Universitário Tabosa de Almeida Asces-Unita, Caruaru, Pernambuco, Brasil.

\section{Risonildo Pereira Cordeiro}

Docente do Centro Universitário Tabosa de Almeida Asces-Unita, Caruaru, Pernambuco, Brasil.

\section{RESUMO}

Introdução. As parasitoses representam um grave problema de saúde pública, nas comunidades mais carentes. Rotineiramente a comida acaba sendo veículo de disseminação de microrganismos como, Entamoeba sp e Giardia sp, também tem destaque a não acessibilidade a tratamentos medicamentosos. Buscou-se analisar a atividade antiparasitária e toxicidade dos extratos: Bidens pilosa, Cucurbita $s p$, Eucalyptus globulus, Mentha piperita L., Ocimum gratissimum, Petroselinum crispum, Allium sativum, Coriadrum sativum, Dysphania ambrosioide, Punica granatum. frente aos cistos da Giardia sp e Entamoeba sp, como potenciais antiprotozoários. Métodos Foram produzidos extratos brutos secos, hidro 
alcoólicos, por método maceração e posterior secagem. Os cistos de Ameba sp. e Giárdia sp., foram expostos em soluções dos extratos nas concentrações de $100 \%, 50 \%, 25 \%$ e 12,5\%, com uma proporção de amostra/extrato (1:10). Foi utilizado a técnica de Artemia salina para observação da toxicidade dos mesmos. Resultados Os extratos de Eucalyptus globulus, Mentha crispa, Allium sativum, Coriadrum sativum e Punica granatum, demonstraram maior espectro de ação frente aos microrganismos. As principais alterações, evidenciadas nos cistos, foram retração da membrana e lise celular com extravasamento do citosol. Observou-se que a maioria dos extratos demonstraram-se praticamente atóxicos. Conclusão As plantas medicinais devem ser estudadas adotando ensaios in vitro e in vivo, para observar a ação antiprotozoária em ambas as situações. $\mathrm{O}$ estudo revelou um potencial para a produção de produtos com fins terapêuticos antiparasitários.

Palavras chaves: Parasitas, plantas medicinais e tratamento.

\begin{abstract}
Introduction. Parasitosis represent a serious public health problem in poor communities. Routinely, food ends up being a vehicle for the dissemination of microorganisms such as Entamoeba sp and Giardia sp. We sought to analyze the antiparasitic activity and toxicity of the extracts: Bidens pilosa, Cucurbita sp, Eucalyptus globulus, Mentha piperita L., Ocimum gratissimum, Petroselinum crispum, Allium sativum, Coriadrum sativum, Dysphania ambrosioide, Punica granatum. against Giardia sp and Entamoeba sp cysts, as potential antiprotozoal agents. Methods Dry, hydroalcoholic crude extracts were produced by maceration method and subsequent drying. Ameba sp. and Giardia sp. cysts were exposed in solutions of the extracts at concentrations of $100 \%, 50 \%, 25 \%$ and $12.5 \%$, with a sample/extract ratio (1:10). The Artemia salina technique was used to observe their toxicity. Results The extracts of Eucalyptus globulus, Mentha crispa, Allium sativum, Coriadrum sativum and Punica granatum, demonstrated a greater spectrum of action against microorganisms. The main alterations, evidenced in the cysts, were membrane retraction and cell lysis with cytosol extravasation. It was observed that most of the extracts were practically non-toxic. Conclusion Medicinal plants should be studied adopting in vitro and in vivo assays, to observe the antiprotozoal action in both situations. The study revealed a potential for the production of products with antiparasitic therapeutic purposes.
\end{abstract}

Key words: Parasites, medicinal plants and treatment.

\title{
1 INTRODUÇÃO
}

Infecções ocasionadas por helmintos e protozoários acometem cerca de 3,5 bilhões de pessoas, sendo a maior parte destas crianças. Sinais e sintomas comumente apresentados são, anemia, dificuldades de crescimento, desnutrição, irritabilidade, retardo cognitivo, aumento da suscetibilidade a outras infecções e complicações agudas são comumente descritos na literatura decorrentes de infecção parasitária1.

As ações necessárias para que se obtenha efetivo controle dessas enfermidades são combinadas de terapêutica, saneamento e conscientização sanitária das pessoas. As crianças e os idosos são os mais acometidos, por estarem frequentemente expostos a constantes condições de reinfecção, quando permanecem em ambientes favoráveis à transmissão².

Dentro do contexto da acessibilidade terapêutica, tornou-se cada vez mais comum o uso de plantas medicinais no tratamento de enfermidades, levando um aumento nos estudos etnobotânicos realizados em 
todas as regiões do Brasil. O maior quantitativo de trabalhos realizados sobre o uso de fitoterápicos, aborda temas sobre o tratamento de doenças em geral, sendo que o uso de plantas com atividade antiparasitária é encontrado como dados secundários nas pesquisas ${ }^{3}$.

Com a elaboração de uma política de âmbito nacional para o uso de plantas medicinais e de fitoterápicos, resultado de um conflito que vem desde a criação do SUS, a implantação da Fitoterapia no Sistema Único de Saúde, facilitou o tratamento da população que não tem acesso a medicamentos. Tendo uma ampla lista de plantas, entre elas apresentando destaque; Allium sativum, Bidens pilosa, Curcuma, Mentha spp, Ocimum gratissimum, Petroselinum sativum, Punica granatum, Eucalyptus globulus e mais 63 espécies vegetais ${ }^{4}$.

O Coriandrum sativum, popularmente conhecida como coentro é uma hortaliça folhosa amplamente utilizada na culinária no Nordeste brasileiro. Apresenta ampla descrição quanto às atividades; antimicrobiana, antioxidante, hipoglicemiante, hipolipidêmica, ansiolítico, analgésica, antiinflamatória 5 .

Allium sativum é uma planta herbácea, considerado um alimento funcional rico em alicina, que apresenta atividade antiviral, antifúngica e antibiótica, um de seus componentes o selênio, descrito na literatura com ação antioxidante. Alguns compostos sulfurados do alho possuem atividade hipoglicemiante, hipotensora, hipocolesterolêmica e antiagregante plaquetária, reduzindo o risco de doenças cardiovasculares ${ }^{6}$.

A Bidens pilosa, pertence à família Asteraceae, com nome popular de picão-preto, esta espécie vegetal originária da América do Sul, encontrada em países tropicais e subtropicais, é amplamente descrita na literatura, sua administração por aplicação tópica. Os compostos majoritários são, acitilênicos e flavonoides, que apresentam funções, anti inflamatória e atividade antimicrobiana ${ }^{7}$.

Nesse contexto, este trabalho estudou a atividade antiparasitária frente aos cistos de Giardia sp e Entamoeba sp,e toxicidade do extrato bruto de 10 plantas medicinais: Bidens pilosa (Picão-preto), Cucurbita sp (Jerimum), Eucalyptus globulus (Eucalipto), Mentha crispa. (Hortelã da folha miúda), Ocimum gratissimum (Manjericão), Petroselinum crispum (Salsinha), Allium sativum (Alho), Coriadrum sativum (Coentro), Dysphania ambrosioide (Mastruz), Punica granatum (Romã).

\section{MÉTODOS}

As matérias primas vegetais foram postas a maceração, em álcool etílico absoluto, durante sete dias, posteriormente os macerados foram filtrados, para obtenção dos extratos brutos fluidos. Em seguida sendo os extratos rota evaporados à temperatura de $60^{\circ} \mathrm{C}$. Após a evaporação de $95 \%$ do álcool, os extratos foram acondicionados em um dessecador a vácuo, até a secura total, quando se obteve os extratos brutos secos dos vegetais. Sendo utilizadas as folhas de Bidens pilosa, Cucurbita sp, Eucalyptus globulus, Mentha 
crispa, Ocimum gratissimum, Petroselinum crispum, Coriadrum sativum, Dysphania ambrosioide, Punica granatum e bulbo do Allium sativum.

Para a realização do bioensaio toxicológico com microcrustáceo Artemia salina, utilizou-se a metodologia descrita por MEYER et al., $1982^{8}$, preparando-se uma solução com sal marinho na concentração de $30 \mathrm{~g} \mathrm{~L}$. O pH foi ajustado entre 8,0 e 9,0, por meio de solução 0,1 mol L-1 de NaOH. Os ovos foram postos para eclodir na solução salina por 48 horas, com aeração constante a $25^{\circ} \mathrm{C}$. Cerca de 12 meta nauplios foram transferidos para tubos de ensaios contendo água do mar e o extrato a ser testado em 6 concentrações diferentes. Os testes foram feitos em triplicata. A contagem dos animais mortos e vivos foi realizada após o intervalo de 24 horas. Utilizou-se também o método de probit, descrita por BLISS, $1935^{9}$, em análise para a obtenção da DL50 e respectivos intervalos de confiança. Os extratos foram considerados ativos a partir da concentração de $50 \mu \mathrm{g} / \mathrm{mL}$.

O isolamento dos cistos foi realizado a partir de amostras fecais cedidas pelo Projeto de Extensão Prevenção e Detecção de Doenças Infectocontagiosas Parasitárias do Centro Universitário Tabosa de Almeida. As amostras foram submetidas à técnica de sedimentação pelo método de Hoffman, Pons e Janer. O isolamento dos cistos de Giardia sp. e Entamoeba sp. seguiu o protocolo de Alvarado \& Wasserman, $2006^{10}$.

O sedimento foi coletado, centrifugado em condições ( $800 \mathrm{xg}, 5$ minutos) e foi lavado três vezes com água. Houve ressuspensão do pellet em $20 \mathrm{~mL}$ de água e dividido em quatro alíquotas de $5 \mathrm{~mL}$ cada. Cada alíquota foi cuidadosamente vertida sobre $3 \mathrm{~mL}$ de sacarose fria $0,85 \mathrm{M}$ e então centrifugada $(600 \mathrm{xg}$, 10 minutos). Observou-se o surgimento de uma película localizada na interface das fases após a centrifugação. Elas foram coletadas, reunidas e homogeneizadas para posterior experimento. O homogeneizado final foi observado ao microscópio, Nikon eclipse E 100, com solução de iodo (1\%) de Dobell e O'Connor para verificação da recuperação dos cistos.

Para o preparo das soluções iniciais, a serem aplicadas no teste antiparasitário, todos os extratos brutos secos foram pesados em $0,05 \mathrm{~g}$, diluídos em $5 \mathrm{~mL}$ de soro fisiológico, estabelecendo diluições nas concentrações de $100 \%, 50 \%, 25 \%$ e $12,5 \%$, em relação a solução inicial.

A exposição dos extratos brutos, frente aos concentrados de cistos (Giardia sp e de Entamoeba sp), teve como controle positivo, $90 \mu \mathrm{L}$ de soro fisiológico e $10 \mu \mathrm{L}$ do concentrado dos cistos com um tempo de exposição de 30 minutos e feita a leitura em microscópio óptico. Seguiram a exposição de $90 \mu \mathrm{L}$ de todos os extratos analisados nas diferentes concentrações acrescidos de $10 \mu \mathrm{L}$ do concentrado dos cistos com tempo de exposição de 30 minutos e feita a leitura com microscópio óptico, observando se houve alterações morfológicas em comparação aos cistos presentes no controle positivo. 


\section{RESULTADOS}

Os extratos brutos secos foram obtidos das folhas, de (Bidens pilosa, Cucurbita sp, Eucalyptus globulus, Mentha crispa., Ocimum gratissimum, Petroselinum crispum, Coriadrum sativum, Dysphania ambrosioide, Punica granatum), e do bulbo, do Allium sativum, como apresentado, Tabela1.

Quanto a toxicidade, CL50, os extratos de Allium sativum, Coriadrum satium, Ocimum gratissimum e Punica granatum, demonstraram-se praticamente atóxico. A Mentha crispa e a Dysphania ambrosioide, apresentaram-se como pouco tóxicas, enquanto que o Eucalyptus globulus, Curcubita sp e Bidens evidenciaram-se moderadamente tóxicas e o Petroselinum crispum expressou-se altamente tóxico. Os extratos e os resultados estão descritos, na Tabela 1 e Imagem 1.

Tabela 1 Espécies, partes utilizadas e toxicidade CL50 das plantas.

\begin{tabular}{|c|c|c|c|c|}
\hline Nome científico & Nome popular & $\begin{array}{c}\text { Parte } \\
\text { utilizada }\end{array}$ & Toxicidade & $\begin{array}{l}C L 50( \\
\mu g / m L)\end{array}$ \\
\hline Bidens pilosa & Picão-petro & Folhas & $\begin{array}{l}\text { Moderadamente } \\
\text { tóxico }\end{array}$ & 311,407 \\
\hline Cucurbira sp & Jerimum & Folhas & $\begin{array}{l}\text { Moderadamente } \\
\text { tóxico }\end{array}$ & 537,478 \\
\hline Allium sativum & Alho & Bulbo & Praticamente atóxico & $1.190,642$ \\
\hline Eucalyptus globulus & Eucalipto & Folhas & $\begin{array}{l}\text { Moderadamente } \\
\text { tóxico }\end{array}$ & 478,365 \\
\hline Mentha crispa & $\begin{array}{l}\text { Hortelã da folha } \\
\text { miúda }\end{array}$ & Folhas & Pouco tóxico & 995,995 \\
\hline Ocinum gratissimum & Manjericão & Folhas & Praticamente atóxico & 787,885 \\
\hline $\begin{array}{l}\text { Petroselinum } \\
\text { crispum }\end{array}$ & Salsinha & Folhas & Altamente tóxico & 284,198 \\
\hline Coriandrum sativum & Coentro & Folhas & Praticamente atóxico & $1.382,6185$ \\
\hline $\begin{array}{l}\text { Dysphania } \\
\text { ambrosioide }\end{array}$ & Mastruz & Folhas & Pouco tóxico & 932,082 \\
\hline Punica granatum & Romã & Folhas & Praticamente atóxico & $2.762,972$ \\
\hline
\end{tabular}

$\mu \mathrm{g} / \mathrm{mL}$; micrograma/ mililitro. 

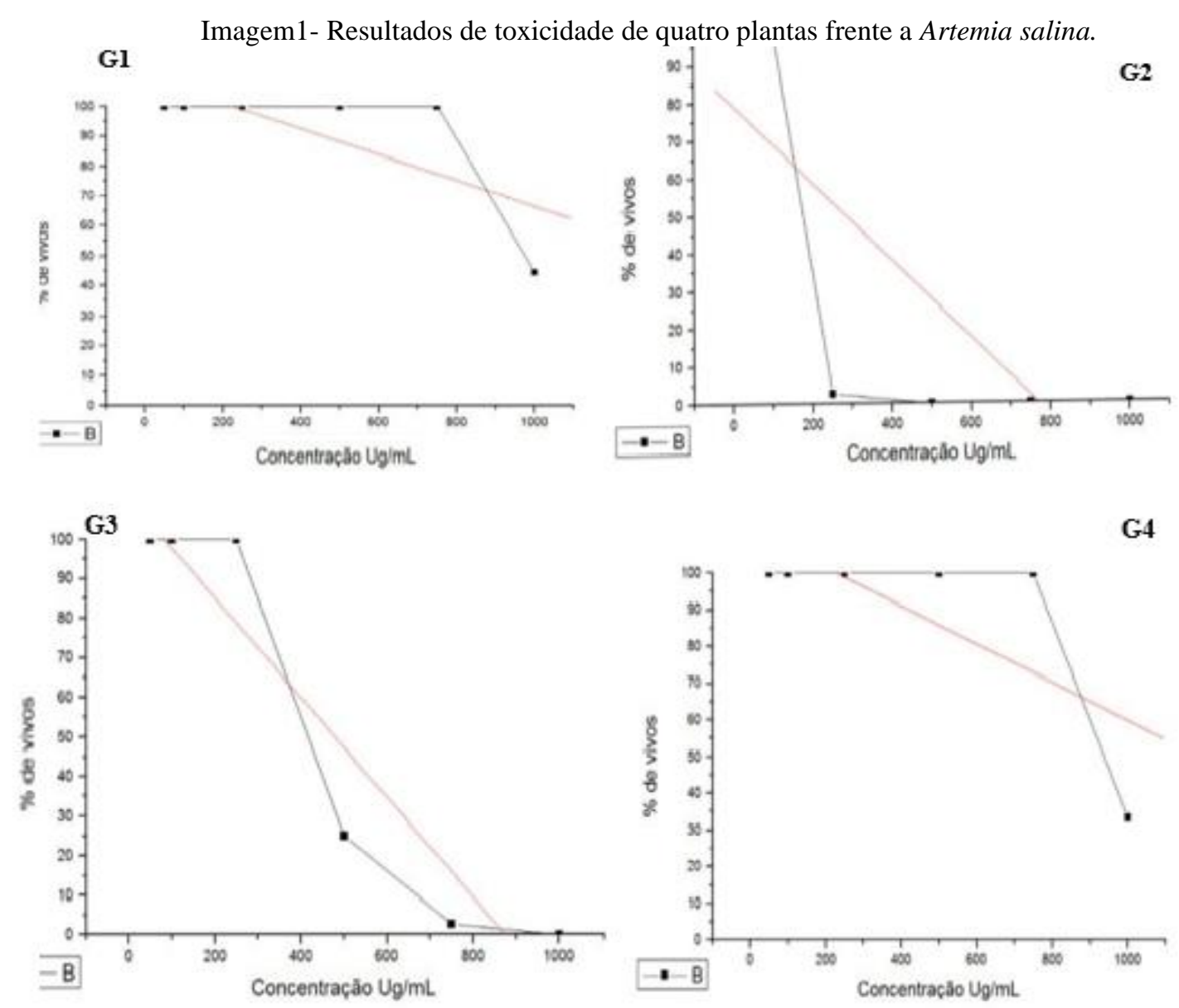

G1- Coentro

G2- Salsinha

G3- Eucalipto

G4- Alho

Após exposição dos protozoários aos extratos foram observadas as alterações morfológicas, comparando-as com a amostra controle, como retração nuclear, extravasamento do citoplasma, vacuolizado e lise celular, foram observadas para as plantas Manjericão, Coentro, Salsinha, Jerimum, Hortelã, Eucalipto, Romã e Alho alterações morfológicas em 50\% dos cistos de Giardia sp analisados. Observou-se alterações morfológicas, em 100\% dos cistos, de ambos os protozoários, para o Manjericão, Coentro, Salsinha. Jerimum, Hortelã, Eucalipto, Romã e Alho. O mastruz não apresentou atividade frente aos protozoários, Tabela 2.

Tabela 2. Atividade antiprotozoária dos extratos frente aos cistos de Entamoeba spp e Giardia spp, intensidade de alterações morfológicas.

\section{Cisto de Giardia sp Cisto de Entamoeba sp}

\begin{tabular}{lllllllll}
\hline $\begin{array}{l}\text { Extrato bruto } \\
\text { seco }\end{array}$ & 100 & 50 & $25 \%$ & $12,5 \%$ & $100 \%$ & $50 \%$ & $25 \%$ & $12,5 \%$ \\
\hline
\end{tabular}




\begin{tabular}{|c|c|c|c|c|c|c|c|c|}
\hline Manjericão & + & + & + & + & - & - & - & - \\
\hline Coentro & ++ & ++ & + & + & + & + & + & + \\
\hline Mastruz & - & - & - & - & - & - & - & - \\
\hline Salsinha & ++ & + & + & + & - & - & - & - \\
\hline Jerimum & + & + & + & + & - & - & - & - \\
\hline $\begin{array}{l}\text { Hortelã da folha } \\
\text { miúda }\end{array}$ & ++ & ++ & ++ & ++ & ++ & ++ & ++ & ++ \\
\hline Picão & - & - & - & - & + & + & + & + \\
\hline Eucalipto & +++ & +++ & +++ & +++ & ++ & ++ & + & + \\
\hline Romã & + & + & + & + & + & + & + & + \\
\hline Alho & + & + & + & + & ++ & ++ & ++ & + \\
\hline
\end{tabular}

(*) Concentrações das diluições dos extratos bruto seco

(-) Sem alterações morfológicas.

(+) Raras alterações morfológicas dos protozoários

(++) Alterações morfológicas em mais de $50 \%$ dos protozoários observados

(+++) Alterações morfológicas em 100\% dos protozoários observados.

A ação evidenciada para o extrato bruto do Eucalipto aos cistos de Giardia sp. apresentou alteração por lise das estruturas da parede dos cistos. Os extratos de, Coentro, Hortelã e Salsinha produziram retração nuclear e lise frente aos cistos de Giardia sp. O Eucalipto, frente aos cistos de Entamoeba sp., apresentaram alterações na morfologia como retração nuclear e lise comparados ao grupo controle. Os extratos de Coentro e Romã, evidenciaram, elevada atividade frente aos cistos de Giardia sp, já para Entamoeba sp., demonstraram pouca ação, Imagem 2 e 3. 
Imagem 2. Controle positivo do Cisto de Giardia sp. e alterações morfológicas dos extratos de Manjericão, Eucalipto e Coentro.

G.a; controle positivo da Giardia

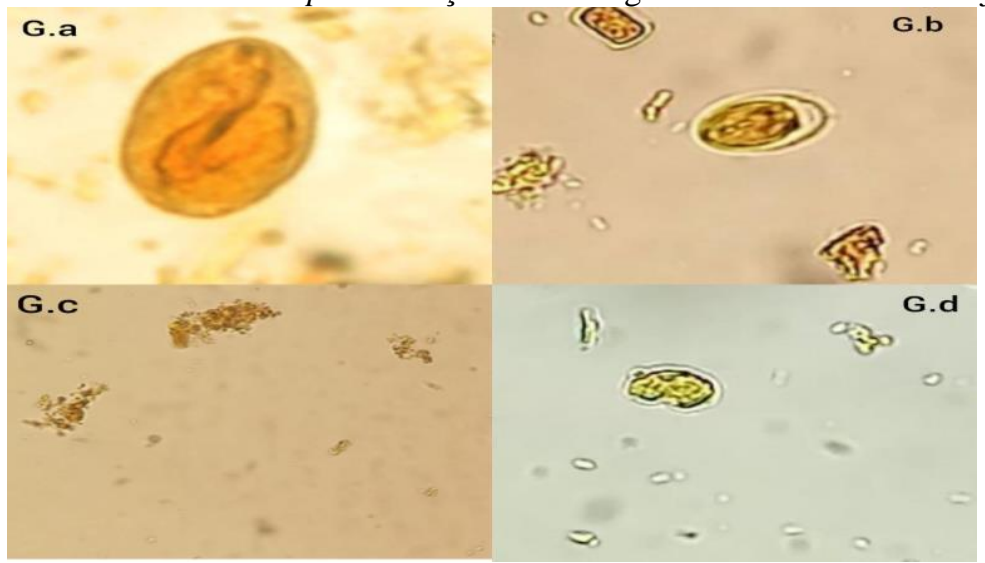

G.b; extrato Manjericão (retração nuclear)

G.c; extrato de Eucalipto (ausência cística)

G.d; extrato de Coentro (lise celular)

Obs:equipamento câmera samsung 24 megapixel.

Imagem 3: Controle positivo do Cisto de Entamoeba sp. alterações morfológicas dos extratos de Eucalipto e Coentro.

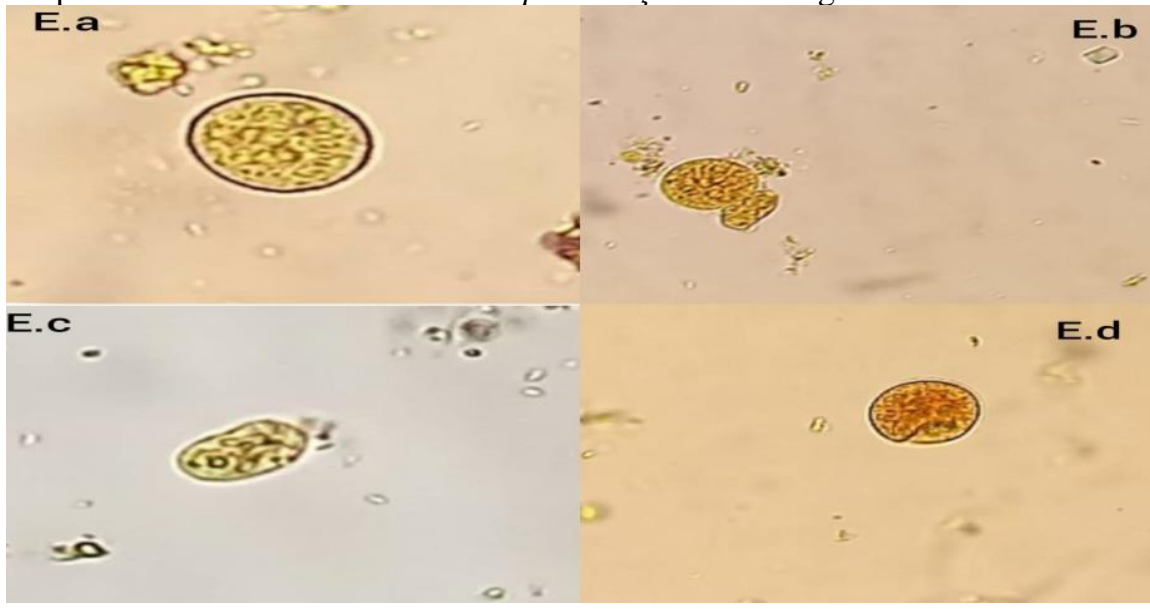

E.a - controle positivo de Entamoeba sp

E.b - extrato e Eucalipto (rompimento da membrana e extravasamento citoplasmática)

E.c - extrato Coentro retração da membrana)

E.d - extrato Eucalipto (rompimento da membrana)

Obs:equipamento, câmera samsung 24 megapixel.

\section{DISCUSSÃO}

Em nossos testes o Eucalyptus apresentou-se moderadamente tóxico; outros estudos realizados com extratos brutos, evidenciaram sua toxicidade por efeitos danosos como gastroenterite, hematúria, taquicardia, miose, dores de cabeça. ${ }^{11}$

Em relação a atividade antiparasitária, o Eucalyptus, expressou elevada efetividade frente aos cistos de Giardia sp. e Entamoeba sp.; com alterações morfológicas e lise de membrana, na totalidade dos cistos de Giardia . Estudos já realizados, com o extratos do Eucalyptus globulus, comprovaram as ações antifúngica, antibacteriana, usado em formulações de inseticidas, acaricida, herbicida, porém não foram relatadas ações frente aos protozoários Giardia sp e Entamoeba sp. ${ }^{12}$ 
O extrato bruto seco de Punica granatum, apresentou-se praticamente atóxico, com baixa atividade antiparasitária frente aos cistos de Giardia sp e Entamoeba.sp, Em trabalhos com extratos brutos foi confirmado a toxicidade, sendo necessário investigações para avaliar genotoxicidade do extrato ${ }^{13}$

A Mentha x piperita, em nosso estudo, caracterizou-se por uma baixa toxicidade, tendo uma atividade moderada em ambos os cistos dos protozoários, observando-se alterações morfológicas por retração de material citoplasmático e rompimento de membrana celular, em metade dos cistos observados. Ensaios com extratos brutos tem revelado uma toxicidade moderada, quando expostos a trofozoítos de Giardia lambria, não apresentou atividade antiparasitária ${ }^{14}$

O extrato bruto do Picão (Bidens pilosa) possui propriedades com potencial antimalárico, em um ensaio in vitro, sendo considerado uma promissora classe para desenvolvimento de fármacos frente a esses protozoários $^{15}$. Contrapondo-se a estudos anteriores, em nosso trabalho a Bidens pilosa, evidenciou moderada toxicidade e ação antiparasitária apenas nos cistos de Entamoeba sp.

A ação antiprotozoária do Allium sativum, foi observada frente aos cistos de Giardia sp. $e$ Entamoeba sp, sendo mais proeminente sua atividade amebicida,,. quanto a toxicidade demonstrou ser atóxico. Alguns estudos científicos expressaram que o bulbo do Allium sativum demonstra baixa toxicidade e boa atividade antiparasitária ${ }^{16}$

O presente estudo observou ação antiparasitária para o Coriandrum sativum, em cistos dos protozoários Giardia sp e Entamoeba sp., obtendo os melhores resultados para os cistos de Giardia sp. O Coriandrum sativum revelou-se atóxico frente as Artêmias salinas. Descrito na literatura, Coriandrum sativum apresentou C150, sendo considerado não tóxico para a técnica da Artemia salina ${ }^{17}$.

O Ocimum basilicum, evidenciou-se ser praticamente atóxico, seu extrato bruto seco, observandose baixa atividade antiparasitária, no presente estudo, frente aos cistos da Giardia sp., sem atividade sobre os cistos de Entamoeba sp.

\section{CONCLUSÃO}

O uso de plantas medicinais, é uma boa alternativa para ajudar no controle de doenças ocasionadas por protozoários gastrintestinais, tendo em vista que as plantas estão inseridas no cotidiano da população, podendo ser utilizadas de maneira preventiva, ou até mesmo complementar a terapia medicamentosa. $\mathrm{O}$ estudo tem seu ineditismo, quanto a ação dos extratos brutos secos de Bidens pilosa, Cucurbira sp, Eucalyptus globulus, Petroselinum crispum, Coriandrum sativum e Punica granatum, sobre as formas císticas infectantes, interrompendo o ciclo evolutivo do microrganismo de maneira profilática. Outro fato importante reside na possibilidade de produção de fármacos antiparasitários, alternativos à população. 


\section{REFERÊNCIAS}

1. Barcelar PAA, Santos JP, Monteiro KJL, Calegar DA, Nascimento EF, Costa FAC. Parasitoses intestinais e fatores associados no estado do Piauí: uma revisão integrativa. Revista Eletrônica Acervo Saúde, v. 10, n. 4, p. 1802-1809, 2018.

2. Godoy EAM. Identificação e genótipos de giardia intestinalis em humanos e animais no noroeste do estado de São Paulo, Brasil. 2012. 143 f. Tese (Doutorado em Medicina Interna; Medicina e Ciências Correlatas) - Faculdade de Medicina de São José do Rio Preto, São José do Rio Preto, 2012.

3. Lima TMS, Santos DRM, Souza RM, Bastos NG, Vannier MAS, Nunes ES, et al. Plantas medicinais com ação antiparasitária: conhecimento tradicional na etnia Kantaruré, aldeia Baixa das Pedras, Bahia, Brasil. Rev. Bras. Pl. Med., Campinas, v.18, n.1, supl. I, p.240-247, 2016.

4. Figueiredo CA, Gurgel IGD, Dantas GGJ. A Política Nacional de Plantas Medicinais e Fitoterápicos: construção, perspectivas e desafios. Physis: Revista de Saúde Coletiva, v. 24, p. 381-400, 2014.

5. Laribi B, Kouki K, M'hamdi M, Bettaied. Coriander (Coriandrum sativum L.) and its bioactive constituents. Fitoterapia, v. 103, p. 9-26, 2015.

6. Fonseca GM, Passos TC, Ninahuaman MFML, Caroci AS, Costa LS. Avaliação da atividade antimicrobiana do alho (Allium sativum Liliaceae) e de seu extrato aquoso. Revista Brasileira de Plantas Medicinais, v. 16, n. 3, p. 679-684, 2014.

7. Borges CC, Matos TF, Moreira J, Rossato AE, Zanette VC, Amaral Bidens pilosa L.(Asteraceae): traditional use in a community of southern Brazil. Revista Brasileira de Plantas Medicinais, v. 15, n. 1, p. 34-40, 2013.

8. Meyer BN, Ferrigni NR, Putnam JE, Jacobsen LB, Nichols DE, McLaughlin JL. Brine shrimp: a convenient general bioassay for active plant constituent. Journal of Medical Plant Research, v. 45, n.1, p. 31-34, 1982;

9. Bliss CI. The calculator of the dosage-mortality curve. Annals of Applied Biologyy, v.22, p. 134$167,1935$.

10. Alvarado ME, Wasserman M. Quick and efficient purification of Giardia intestinalis cysts from fecal samples. Parasitology Research, 99(3), 300-302, 2006

11. Arauz ADM, Jiménez JCU, Flores BOM. Determinación de DL50 aplicando bioensayo con Artemia Franciscana, en las especies vegetales Citrus aurantium, Ruta Chalepensis, Eucalyptus camaldulensis laboratorio de química, UNAN MANAGUA, 2017.

12. Pereira JL. Composição química dos óleos essenciais de espécies de Eucalyptus L'Herit (Myrtaceae). Universidade Federal de Viçosa, 2010.

13. Dienstmann ERB. Plantas com indícios de toxicidade utilizadas como medicinais no Rio Grande do Sul, Brasil. 2011. 
14. Brasil. Monografia da espécie Mentha $x$ piperita L. (Hortelã Pimenta). Ministério da Saúde e Anvisa. Brasília, 2015

15. Tobinaga S, Sharma MK, Aalbersberg WGL, Watanabe K, IguchI K, Narui K. Isolation and Identification of a Potent Antimalarial and Antibacterial Polyacetylene from Bidens pilosa. Planta Medica, 75(06), 624-628, 2009

16. Krishnaraju AV, Rao TV, Sundararaju D, Vanisree M, Tsay HS, Gottumukkala VS. Biological screening of medicinal plants collected from Eastern Ghats of India using Artemia salina (brine shrimp test). Int. J. Appl. Sci. Eng, v. 4, n. 2, p. 115-125, 2006.

17. Lopez MAT, Tovar GM, Mendez IMC, Lujan CG, Valenzuela COP, Menchanca MCV, et al. Evaluation of the chelating effect of methanolic extract of Coriandrum sativum and its fractions on wistar rats poisoned with lead acetate. African Journal of Traditional, Complementary and Alternative Medicines, v. 14, n. 2, p. 92-102, 2017. 